\title{
Inspection of HVOF-coated Pelton wheels using active thermography
}

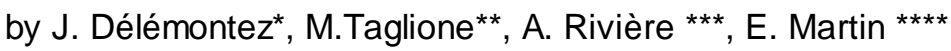 \\ * Électricité de France, Division Technique Générale, 38040 Grenoble, France, jerome.delemontez@edf.fr \\ ${ }^{* *}$ AREVA NDE Solutions France, 71100 Chalon sur Saône, France, matthieu.taglione@areva.com \\ ***Électricité de France, UP Alpes 38040 Grenoble, France, alice.riviere@edf.fr \\ ${ }^{* * *}$ Électricité de France, Ceidre 93 Saint-Denis, France, etienne.martin@edf.fr
}

\begin{abstract}
EDF is performing an experimental study aimed at covering Pelton wheels with hard coating HVOF (High Velocity OxyFuel), which would provide a better abrasion resistance of the wheels. The need for detecting underlying defects and the lack of sensibility of traditional MT (Magnetic particle Testing) in this case made EDF look at others non destructive examination techniques.
\end{abstract}

Feasibility tests were at first led using representative mock-ups and an active thermography inspection system developed by AREVA NDE Solutions called the Photo-Thermal Camera (PTC). Further tests were then realized on real Pelton wheels under fully realistic conditions.

Keywords : Pelton, HVOF coating, MT replacement, inspection, thermography, crack.

\section{Context}

Among the many hydroelectric plants operated by the EDF Alps Production Unit, some facilities are distinguished by the use of turbine water from melting glaciers. This water, which contains a lot of sediment and mineral particles, causes a phenomenon of strong wear by abrasion of the Pelton turbines used in these installations, given their intensive use in run-of-river power stations (e.g. power plants in the French alps with a drop height of around $400 \mathrm{~m}$, four Pelton double turbines each producing yearly about $300 \mathrm{GWh}$ ).

So far, in order to prevent and limit this wear phenomenon that can lead to major damage, the buckets of the Pelton wheels concerned have been treated (excluding the attachment area) with traditional metalizing coatings (type TAFA $28 \% \mathrm{Cr}$ coatings) applied using flame or arc welding, techniques with which the EDF repair workshops are perfectly familiar and competent. These techniques make it possible to leave thick deposits, which can compensate for high wear values, and to use these wheels until they are scrapped.

Before the first coatings were used, the average operating performance was 4 years with a loss of half of the thickness of the buckets, thus requiring major workshop repairs. This maintenance strategy using anti-wear coatings made it possible to extend the life of the Pelton wheels by 2 years in these facilities.

To improve operating performance of the wheels subject to wear, the EDF Alps Production Unit decided in early 2009 to experiment with HVOF (High Velocity Oxy-Fuel) coatings deposited by hypersonic flame on the Pelton wheel buckets. This technique, which had already been used by EDF on other components for years, makes it possible to deposit a very hard material (tungsten carbide $86 \% \mathrm{WC}$ ), which is therefore highly resistant to abrasion, with the best grip characteristics of the coating at a very fine thickness (around $300 \mu \mathrm{m}$ ).

In February 2009, new wheels coated using the HVOF procedure were fitted to a turbine in each of three "pilot" plants. The feedback from 10,000 hours' operation has already indicated a drastic improvement in the state of wear of the coatings and allows strong supposition that there could be a gain of a further 2 years of useful life compared with traditional coatings, see figure 1:

- $\quad$ HVOF-coated buckets have a very smooth surface with hardly any wear marks ; 
- $\quad$ the coating is still present in practically all functional areas with little loss of thickness ;

- $\quad$ some spalling of the coating can be seen, particularly on the median edges and in the cutouts but there was little noticeable change between inspections.

The only negative point is the transition area between the coated part and the substrate in the area where the bucket is attached, which could cause premature differential wear in this area. The attachment area was deliberately left uncoated in order to be able to carry out periodic Non Destructive Examinations (NDE) by magnetic particle inspection (MT) to detect the presence of cracks in this area subject to heavy fatigue, in accordance with the EDF maintenance doctrine.

NDE by magnetic particle testing (MT) carried out by the EDF maintenance teams is based on the search for the indication of a crack caused by a phenomenon of flux leakage from the bucket that has been magnetised and sprayed with a developer containing fine ferro-magnetic particles. The HVOF anti-wear coating acts as a barrier against the phenomenon of flux leakage. The main consequence of this is a strong reduction in the sensitivity of detection as illustrated in figure 2, which is unacceptable in areas subject to high strain from fatigue. Failure to detect a crack several millimetres long during a periodic inspection could in fact lead to breakage and ejection of a bucket in service, which is unacceptable in terms of risk to property and people.

Furthermore, EDF does not at present have any NDE solution for checking the quality of the HVOF coatings made available by its suppliers: for example, disbonds between the coating and the wheel that could lead to HVOF coating loss cannot be detected during pre-service or in-service inspection.

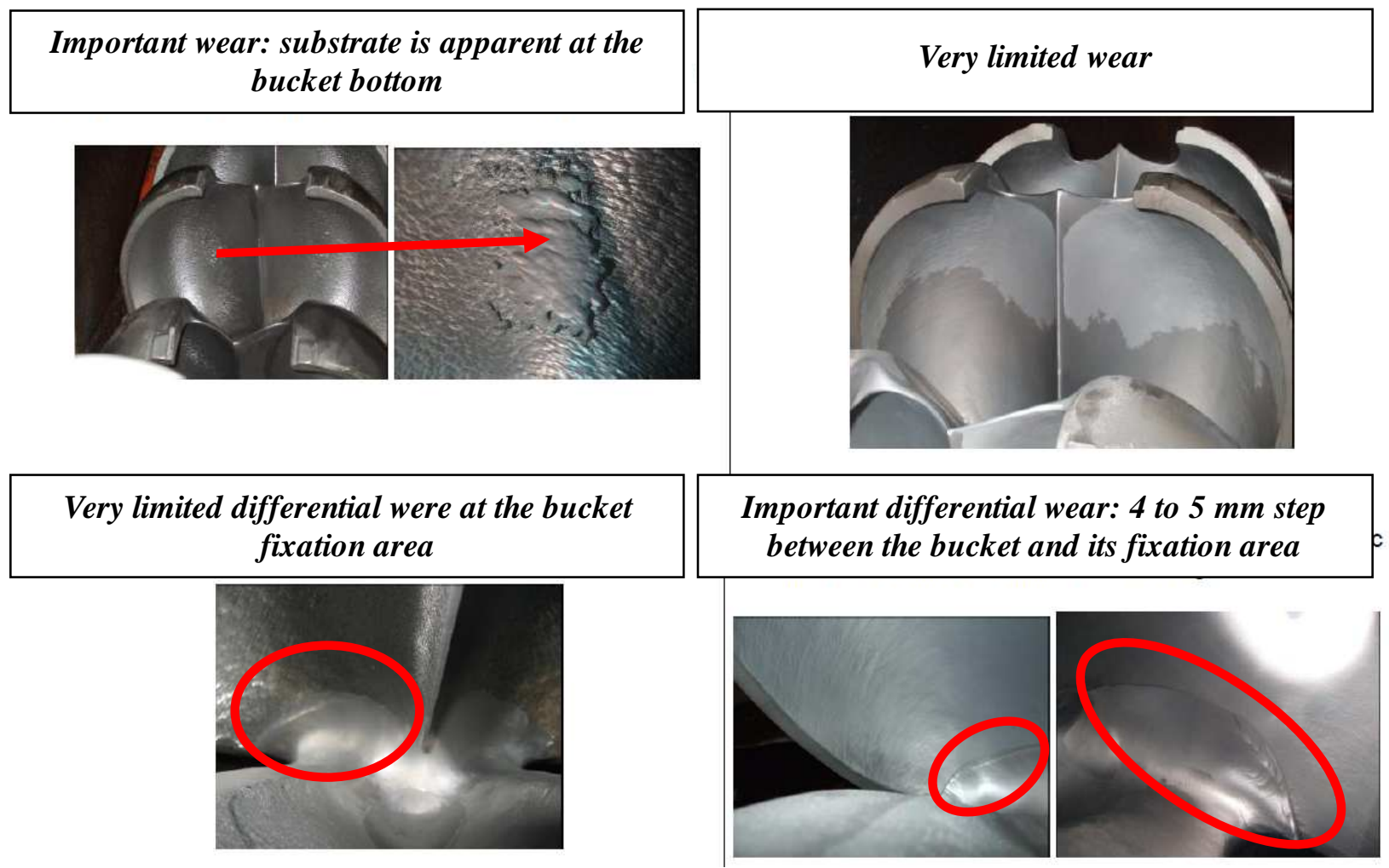

Figure 1: Comparison of wear state of the two types of coating tested (left: arc-welding TAFA, right: HVOF)

To allow the Alps Production Unit to attain the target of doubling the total lifespan of the wheels by applying a HVOF type coating to the whole surface of the buckets (including the attachment area), the EDF General Technical Division (DTG) has proposed studying the feasibility of NDE by active thermography. To do this, the EDF DTG in agreement with the Alps Production Unit has developed a test programme to evaluate the feasibility of cracks and disbond detection under the HVOF coating. EDF DTG has asked AREVA NDE Solutions to evaluate the Active Photothermal Camera (PTC), a laser thermography prototype inspection tool. The PTC seems currently to be the most developed alternative solution to magnetic particle inspection and penetrant testing as already evaluated for some nuclear power plant applications with EDF- Ceidre. 

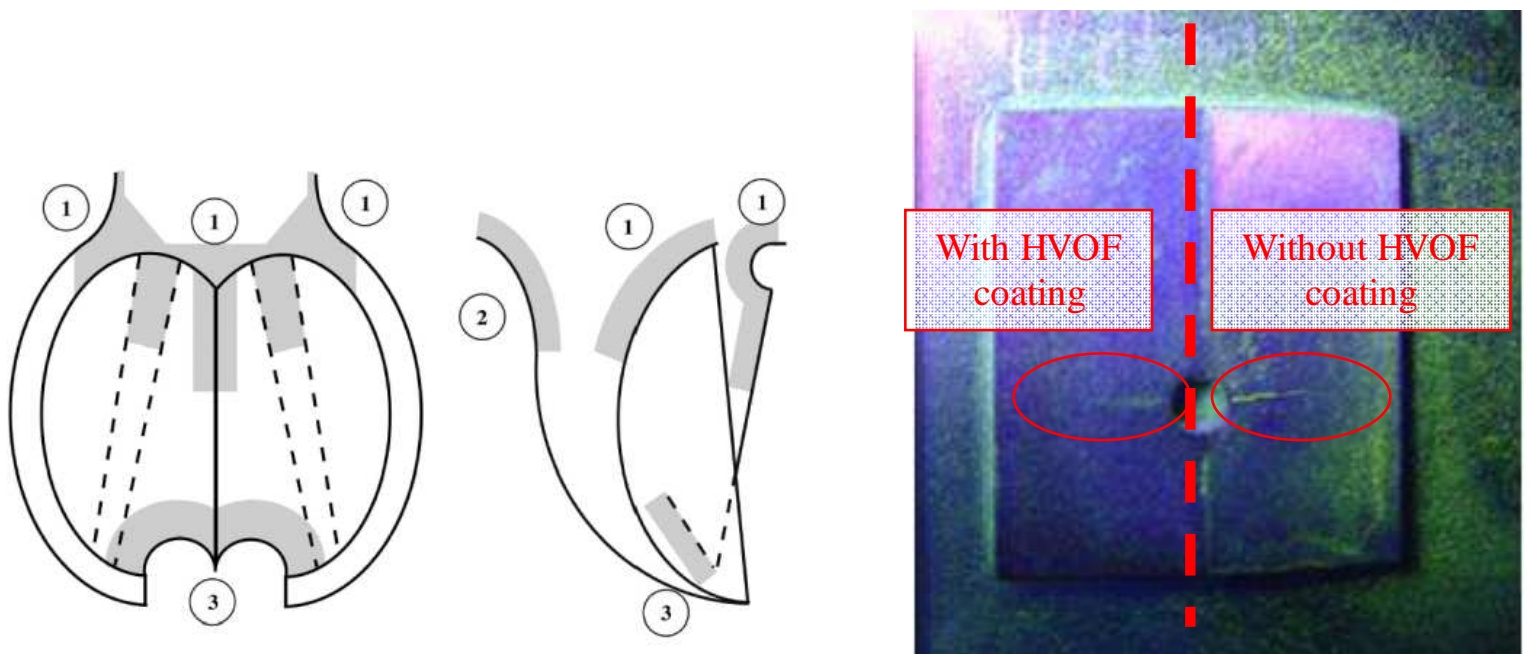

Figure 2: Areas sensitive to cracking in Pelton buckets - Effect of the presence of the HVOF coating on the detection sensitivity of the NDE by MT

\section{Thermography in Non-Destructive Examination (NDE)}

\section{Introduction}

Thermography is the measurement of the temperature at different points of a body. The analysis of the spatial and/or temporal evolution of the temperature set obtained gives information about this body's material, integrity or even geometry.

A very simple application of thermography is measuring the temperature of electrical connections: due to the Joule effect, a loose connection will produce more heat than a well-fit one, and it will be easy to differentiate them. Identically a shaft bearing with improper lubrication will heat up more than it should when rotating; this will indicate improper operating conditions. Here, the parts under inspection generate the temperature elevation, during standard operation. The inspection system does not have to generate heat: this is called passive thermography. A major drawback of this family of techniques is that the inspection possibilities are limited to some typical cases such as those listed above where the anomaly generates the temperature change.

If we want to use heat-flow or change in temperature to inspect parts at rest or parts that do not produce heat, the inspection system has to induce heat into the part. Two different types of methods can achieve this:

- methods that induce global or local heat in the part bulk. The analysis of heat diffusion within the part can provide an indication on the part's local heat conductivity. This conductivity can be affected by discontinuities, thickness variation, material non-homogeneity.... Typically hot or cold fluids (air, water...), light, laser, electrical conduction or induction heating are used for this purpose;

- $\quad$ methods that generate heat directly at the location of a discontinuity thanks to the Joule effect or to mechanical effects such as friction. The defects will behave as heat sources, which the inspection system will detect. These methods can only detect discontinuities such as cracks, delaminations, lack of fusion.... Typically, vibration, electrical conduction or induction heating are used for this purpose.

The detection of heat patterns in inspected parts can be realized in different ways, some of which are contact-less. As explained by Planck's law, every body with a temperature above $0^{\circ} \mathrm{K}$ will emit infrared radiation. The infrared spectral density will depend only on the body temperature in the ideal case. An infrared camera can hence be used to obtain the accurate temperature of an object. Nowadays, infrared cameras have up to $1024 \times 1024$ pixels and reach a thermal resolution in the $0.01^{\circ} \mathrm{K}$ range. This means that a rise of only a few degrees of the surface inspected is enough to allow detection of the thermal flux perturbations over a wide area with high spatial resolution. This allows applying active thermography solutions for NDT applications. 
In reality, the infrared emission will depend not only on Planck's law but also on the emissivity of the part inspected which is a function of the material, surface condition, observation angle, etc. Different techniques for correcting for this emissivity factor exist. One that is useable for inspecting metals using laser heating was developed and patented by AREVA NDE solutions.

\section{The Photothermal Camera (PTC)}

As explained above, if we want to inspect a part that does not produce heat itself, the inspection system must induce heat into the inspected part. A very convenient way of doing so uses light: there is no need of contact with the part, and the power delivered does not vary with the distance if afocal lighting is used. A properly shaped laser fulfils this condition with high light power density and is the basis of the Photothermal Camera prototype. An infrared camera records simultaneously the evolution of the heat generated in the part using this laser light. As explained above, this allows the detection of many features of the inspected part.

The emissivity of the part inspected should not affect the temperature measurement. However, on real parts it varies significantly according to many factors. One way to correct for its influence is scanning the part inspected two times: forward and backwards. The impact of emissivity on the acquired images will be the same for both the forward and the backwards images; however, the signal due to the disturbances in heat propagation will be reversed. A subtraction of the forward and the backward images will cancel out the emissivity signal leaving only the image information due to the perturbation of the heat flux. Analysis of data acquired this way is straightforward.

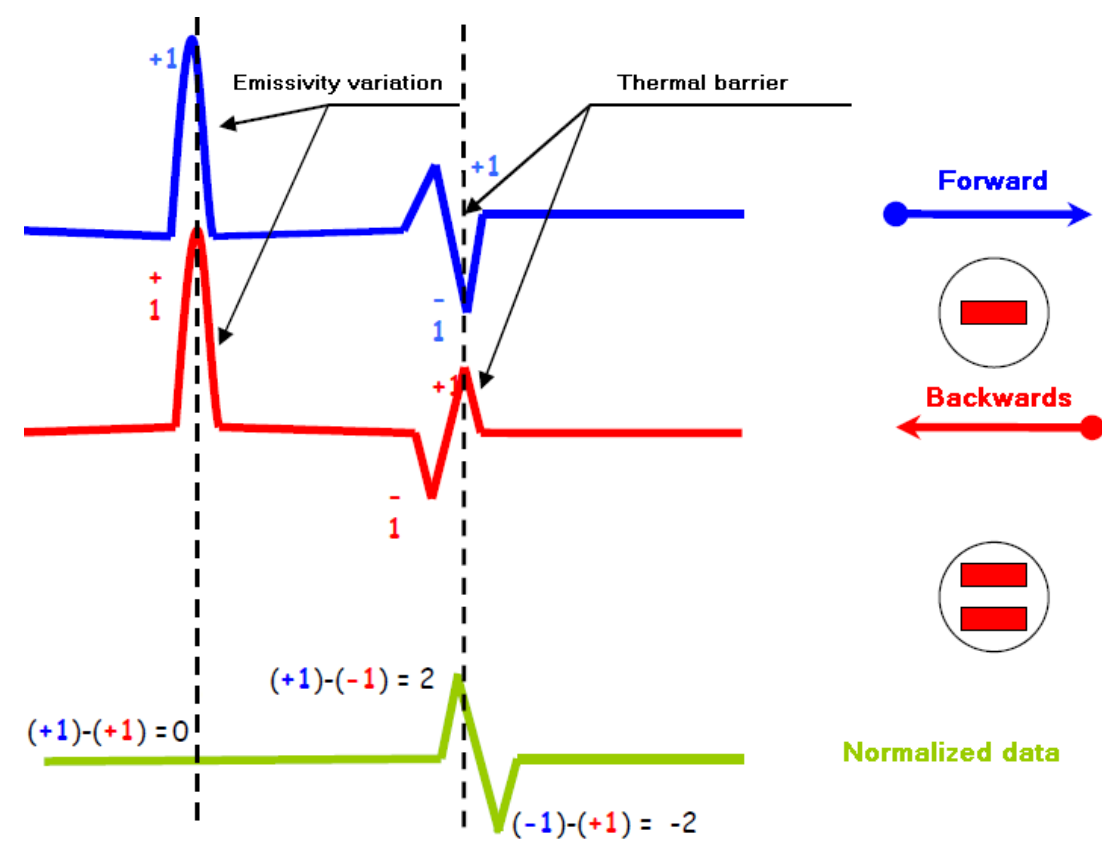

Figure 3: Photothermal camera normalization principle

\section{Thermography vs. MT}

The comparison of thermographic inspection using the PTC prototype and magnetic particle testing can be realized using the calibration samples from the existing EDF procedure. This calibration sample is designed according to EN 9934-2. It comprises surface breaking fatigue cracks, as can be seen on Figure 4.

Using laser thermography, we can see that the crack networks can be detected the same way they are with magnetic particle testing. This concurs with the experience AREVA NDE Solutions has with this technique, which shows that its sensitivity is in the same range as MT for both surface breaking and underlying cracks on steel components. 

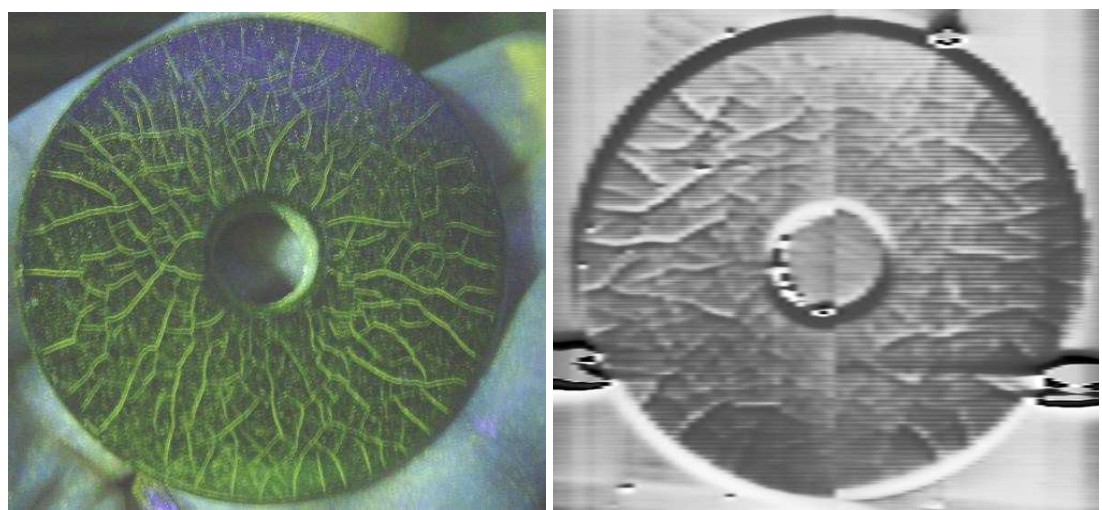

Figure 4: Comparison of MT (left) vs. PTC prototype (right)

\section{LASER thermography of HVOF-coated Pelton wheels}

\section{Experimental approach}

This development followed a progressive approach defined by EDF DTG. First, the inspection feasibility was determined at AREVA NDE Solution's Technical Centre (NETEC), on mock-ups representative of HVOF-coated Pelton wheels with different kinds of defects (EDM, surface breaking and underlying cracks, disbonds). Real wheels with a fatigue crack and cladding-cracks in the bucket fixation area were then inspected; both sound and defective bucket fixation areas were coated with HVOF for comparison of results. Subsequently, the inspection system was brought into a realistic environment to perform an inspection at the Passy dam in the French Alps.

\section{Preliminary experiments}

All preliminary tests were conducted on a CrNi17-4 martensitic stainless steel mock-up covered with a $300 \mu \mathrm{m}$ thick HVOF layer. This mock-up comprises one surface breaking EDM notch, four underlying EDM notches, and one flatbottom hole. The defects characteristics are listed in the table below:

\begin{tabular}{|c|c|c|c|c|}
\hline Defect number & Length $(\mathrm{mm})$ & Depth $(\mathrm{mm})$ & Ligament $(\mathrm{mm})$ & $\begin{array}{c}\text { Orientation to the } \\
\text { mock-up surface }\end{array}$ \\
\hline 1 & 10.0 & 1.0 & 0.0 & Perpendicular \\
\hline 2 & 5.0 & 1.0 & 0.30 & Perpendicular \\
\hline 3 & 2.0 & 1.0 & 0.30 & Perpendicular \\
\hline 4 & 45.0 & 5.0 & 0.48 & Perpendicular \\
\hline 5 & 10.0 & 0.22 & 0.63 & Parallel \\
\hline 6 & 6.0 & 30.0 & 0.67 & Parallel \\
\hline
\end{tabular}

Inspection of this mock-up showed that under proper operating conditions, all the defects embedded into the mock-up are detected using thermographic inspection. Discontinuities perpendicular to the surface stand for cracks and are detected thanks to the typical signal they provide, as explained above. Discontinuities parallel to the surface stand for disbonds and are detected because of the important heat elevation at their location: heat cannot dissipate into the bulk of the part, and stays in the coating thereby generating a temperature rise.

The indications linked to defects 2 and 3 are extremely small, but could be detected by the inspection system provided the right experimental conditions are used. For this particular application, the inspection system is able to detect cracks with length down to $2.0 \mathrm{~mm}$.

The smallest disbond that was present on the mock-up was seen: for this particular application, the inspection system is able to detect disbonds with diameter down to at least $6.0 \mathrm{~mm}$. 
applications with EDF Ceidre - regarding the sensibility of detection:

- $\quad$ distance between PTC and object (500 to $2000 \mathrm{~mm}$ );

- $\quad$ disorientation of object and defect $\left(+/-45^{\circ}\right)$;

- $\quad$ scan speed (2.5 to $15 \mathrm{~mm} / \mathrm{s})$;

- Integration time;

- etc....
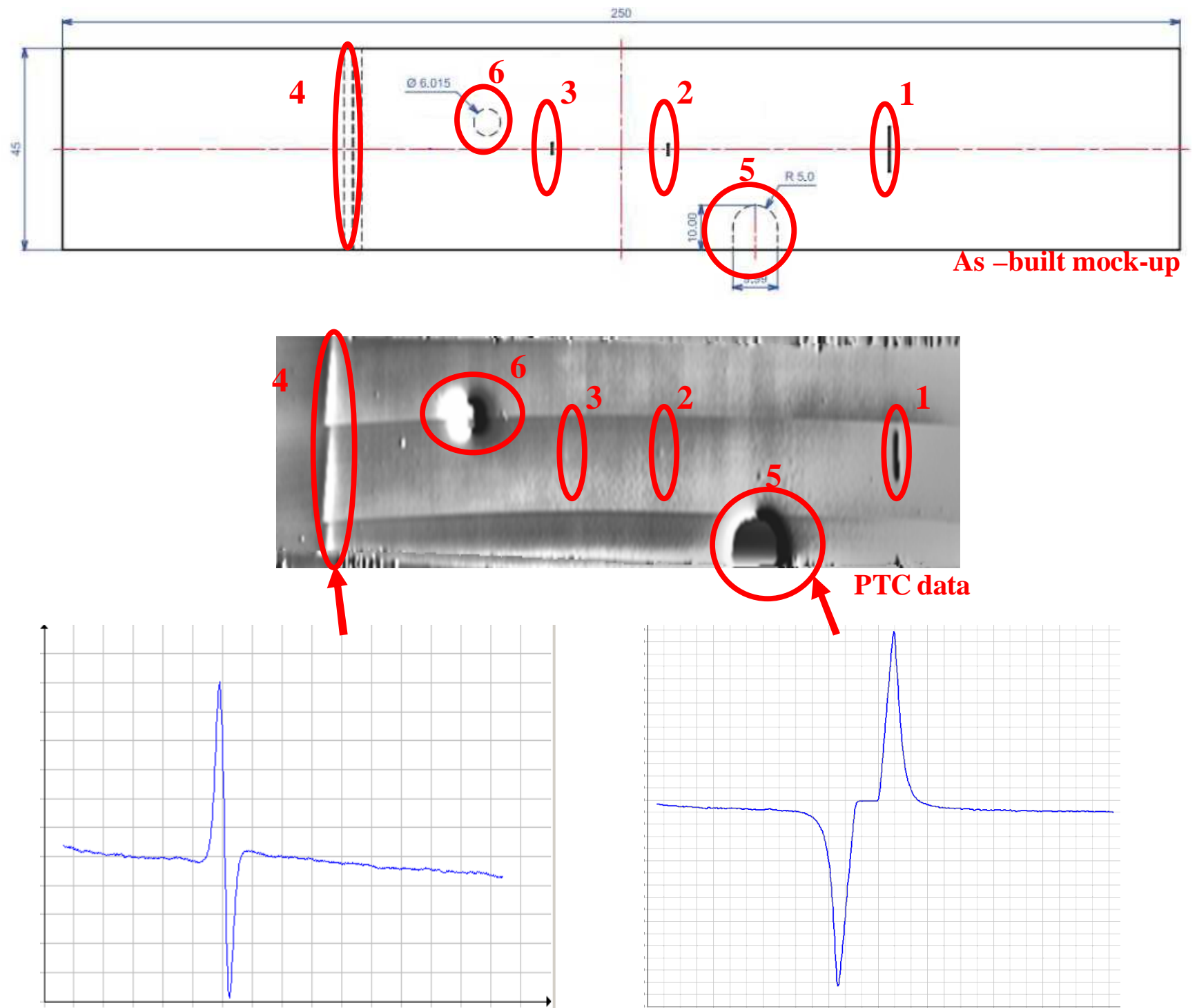

Figure 5: Results of the preliminary tests led at AREVA NDE Solution Technical Centre (arbitrary units) Top : mock-up drawing and associated indications

$$
\text { Bottom left: indication of a discontinuity }
$$

Bottom right: indication of a disbond

\section{Inspection of defectives wheels}

During a periodic inspection, a fatigue crack was found on the bucket fixation area of an uncoated Pelton wheel from the Passy dam. The wheel was dismissed and made available for NDE training and testing. The defect area, as well as a sound bucket-fixation area on a nearby bucket, were coated with HVOF. The PTC prototype was placed in front of the wheel as can be seen on Figure 6, and both areas were inspected in blind-test conditions from this position at a $1 \mathrm{~m}$ distance. The results obtained and shown on Figure 7 clearly indicate a discontinuity in one of the HVOF-coated bucket 
fixation areas, while the other is sound: this corresponds to what had been found using MT before coating.

The crack dimensions and localisation were measured from the thermography data, and were found to be within $10 \%$ of the MT results. Those tests were led in Albertville, at an EDF maintenance facility; the next step planned was conducting tests in a real hydropower plant.
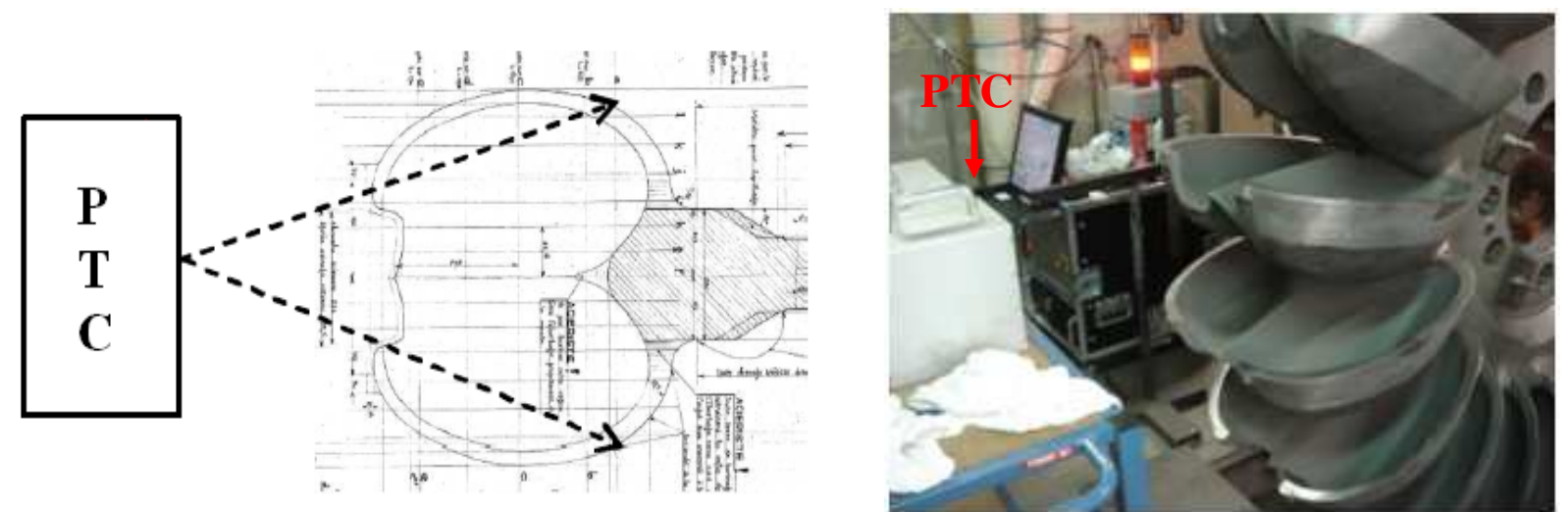

Figure 6: PTC position for bucket fixation area inspection
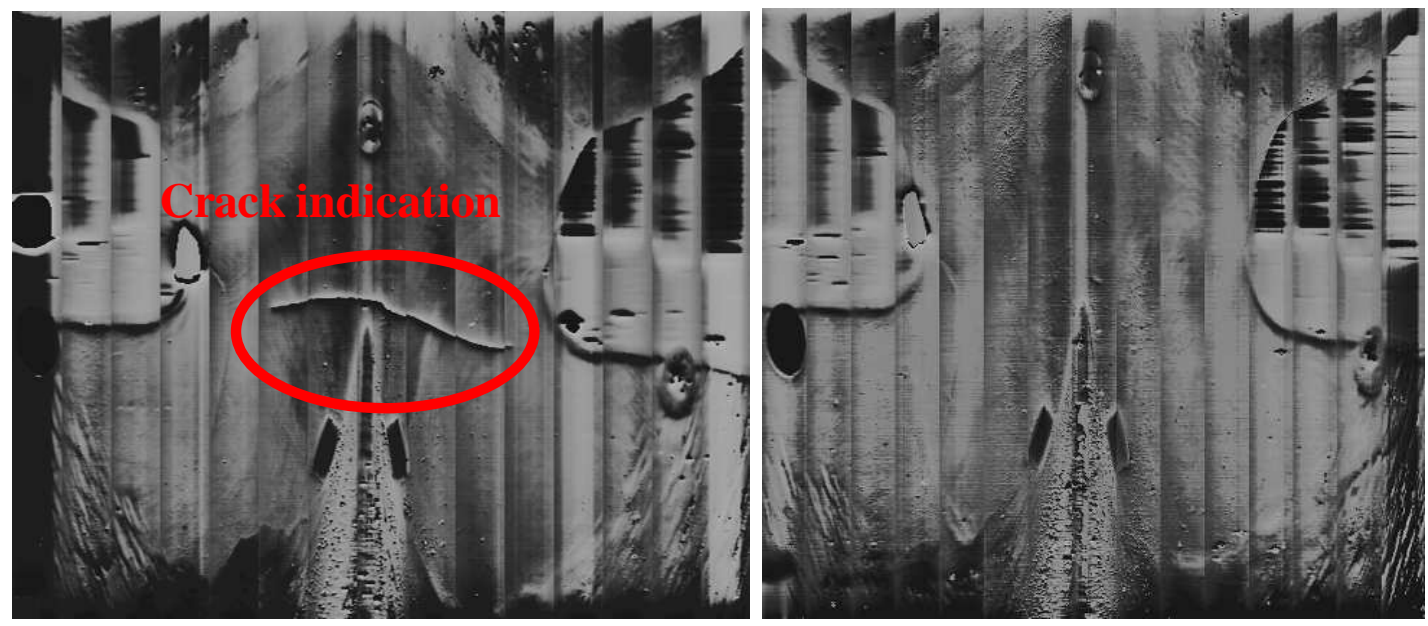

Figure 7: Passy wheel inspection using the PTC prototype

Left: cracked bucket fixation area

Right: sound bucket fixation area

Another example was the PTC inspection of a new Pelton Wheel from Les Bois power plant which buckets were coated in two steps with HVOF. Results showed some crack indications with a clear thermal barrier signature at the interface of the two HVOF layers. A PT examination was performed and confirmed the presence of surface breaking cracks but with a weak contrast because of the surface condition of the coating, as illustrated on Figure 8. 

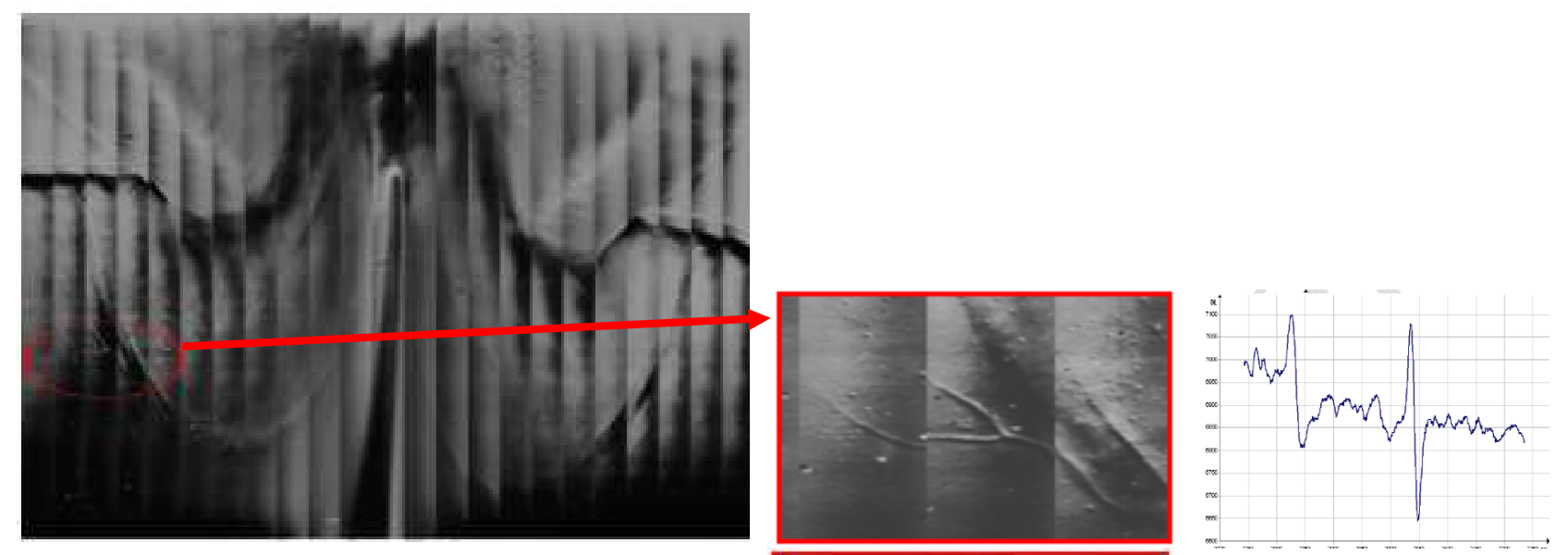

Figure 8: Les Bois wheel inspection using the PTC prototype

Top : thermal barrier indication at the interface between two layers of coating

Bottom : surface breaking crack indication with PT examination

\section{Onsite wheel inspection}

The Passy power plant has one Pelton wheel coated with HVOF (except the attachment area). During a planned maintenance, which included MT inspection, one of the wheels underwent PTC inspection. Neither the MT inspection nor the PTC inspection showed any indication of cracking on this wheel.

This allowed the evaluation of the potential environmental interactions during an inspection in a real hydropower plant, and provided data about realistic implementation of this inspection in terms of positioning, control rate and safety.

The positioning of the camera for inspecting the areas described on Figure 2 requires a wide working range because of the various geometries that are at stake. Because laser heating is afocal, and because the PTC is designed to work from $10 \mathrm{~cm}$ up to $200 \mathrm{~cm}$ of the inspected surface, all required areas could be easily accessed, see Figure 9.

The inspection rate of the demonstration system used was rather slow (30 minutes per inspected area), as it is designed to inspect over a $25 \mathrm{~mm}$ width. In the present case here, it would be advisable to use a larger laser line: for example a $100 \mathrm{~mm}$ line would reduce the inspection time by a factor of four.

Last but not least, the laser used for performing the heating being a class IV laser its use requires to protect the users from its rays. A safety tarp was set around the acquisition area, and providing an efficient safety light barrier exclusion zone. Another team was working very close to the inspected wheel (see white barrier on Figure 9), and presented no issue during the whole inspection duration. 

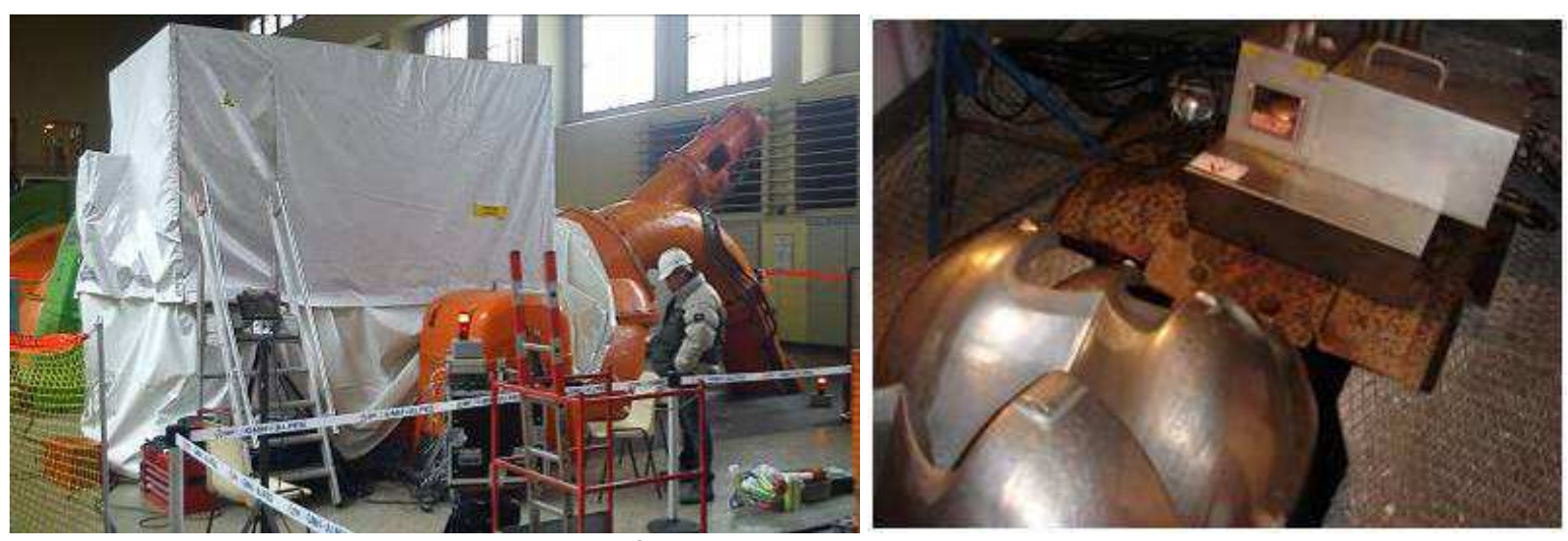

Figure 9: prototype PTC under laser protection tarp during Pelton wheel inspection at Passy

\section{Conclusion}

EDF is attempting to implement a hard coating of Pelton wheels subject to abrasion mainly from sediments carried by meltwater, targeting a wheel life extension by up to a factor of two. The coating used is HVOF (High Velocity Oxy-Fuel); it was applied to buckets on different wheels and proved to be a promising process. However, this coating does not allow the usual magnetic particle (MT) non-destructive examination (NDE) of the wheel as the sensitivity of MT is very low on coated parts.

AREVA NDE Solutions has developed an inspection tool based on LASER active thermography, and which does not have the limitations of MT. This prototype tool, called the Photothermal Camera, allows contactless inspection of metals for different types of defects, such as surface-breaking or sub-surface fatigue cracks and disbonds.

Preliminary tests conducted at the AREVA NDE Solutions Technical Centre (NETEC) and EDF facilities using the Photothermal camera prototype allowed the detection of all defects defined by EDF into mock-ups as well in real components including surface breaking and under coating flaws, parallel or perpendicular to the surface.

The successful realization of this inspection in a real environment proved the possibility of a routine use of thermography for preventive maintenance of HVOF-coated Pelton wheels. Furthermore, experimental studies of the essentials parameters shows that it was possible to qualify this new NDT solution according highest standards such as European ENIQ and French R-SEM qualification.

Following these successful tests, the improvement and industrialisation of the prototype PTC is now acted in form of a subsidised project with other industrial partners. The expectation is to obtain an industrial PTC with improved capacities regarding inspection duration and portability and to bring it to the same level as standard NDT techniques. 